in vivo $34: 3601-3610(2020)$

doi:10.21873/invivo.12205

\title{
Comparison of the Outcome of Patients Protected by the Wearable Cardioverter Defibrillator (WCD) for $<90$ Wear Days versus $\mathbf{2 0 0}$ Wear Days
}

\author{
TOBIAS C. DREHER ${ }^{1 *}$, IBRAHIM EL-BATTRAWY ${ }^{1,2^{*}}$, SUSANNE RÖGER $^{1,2}$, \\ STEPHANIE L. ROSENKAIMER ${ }^{1,2}$, STEFAN GERHARDS ${ }^{1,2}$, JÜRGEN KUSCHYK ${ }^{1,2}$, \\ MARTIN BORGGREFE ${ }^{1,2}$ and IBRAHIM AKIN ${ }^{1,2}$ \\ ${ }^{1}$ First Department of Medicine, Faculty of Medicine, University Medical Centre Mannheim (UMM), \\ University of Heidelberg, Mannheim, Germany; \\ ${ }^{2}$ German Centre for Cardiovascular Research (DZHK), Partner Site, Heidelberg/Mannheim, Germany
}

\begin{abstract}
Background/Aim: The wearable cardioverter/ defibrillator (WCD) is recommended to prevent sudden cardiac death (SCD). Guidelines suggest a 90 days' period, but prolongation of WCD wear time until increasing the ejection fraction ( $\geq 35 \%)$ might be suggested. Patients and Methods: A cohort of 153 patients with prescribed WCD were divided into two groups: $A<90$ wear days' group $(n=112)$ vs. $\geq 90$ wear days' group $(n=41)$ and followed. Results: In the first group, WCD shock occurred in $3.6 \%$ of patients, $47.3 \%$ improved in left ventricular ejection fraction (LVEF) after 3 months, and $37.5 \%$ had a cardiac implantable electronic device (CIED) implantation with appropriate implantable cardioverter defibrillator (ICD) shock events occurring in 6 patients. Two of these patients already received WCD shock therapy due to ventricular fibrillation. A $20.5 \%$ improved in LVEF after 6-12 months, but $73 \%$ were already implanted with ICD. In the second group, $4.9 \%$ received WCD shock, $34.1 \%$ improved in LVEF after 3 months, $48.8 \%$ were implanted with ICD, and 2 had ICD shocks during follow up time. LVEF improvement after 6-12 months occurred in 26.8\%. ICD implantation was prevented in $7.3 \%$ of patients due to LVEF recovery. Conclusion: Prolonging wearing days of WCD may reduce the number of inappropriate ICD implantation.
\end{abstract}

This article is freely accessible online.

*These Authors contributed equally to this study.

Correspondence to: Tobias C. Dreher, First Department of Medicine, University Medical Centre Mannheim, University of Heidelberg, Theodor-Kutzer-Ufer 1-3, 68167 Mannheim, Germany. Tel: +49 6213831447, e-mail: tobias.dreher@stud.uni-heidelberg.de

Key Words: Wearable cardioverter defibrillator (WCD), heart insufficiency, sudden cardiac death.
The Wearable cardioverter defibrillator (WCD) may be considered for patients with low $(<35 \%)$ left ventricular ejection fraction (LVEF) who are at risk of sudden cardiac death (SCD), but implantable cardioverter defibrillator (ICD) implantation is not yet indicated (1). These can be patients with transient or unknown risk for ventricular tachyarrhythmia and $\operatorname{SCD}(1,2)$.

Because many patients recover after diagnosis of heart failure with severely low LVEF $<35 \%$ and high risk of SCD, WCD is most commonly prescribed for 90 days (3-5). During this time, physicians should evaluate heart failure therapy and treat patients with optimal medical therapy (OMT) according to current European Society of Cardiology (ESC) guidelines for heart failure (6). Different studies have shown that early ICD implantation might not improve the outcome of patients with ischemic (ICM) and non-ischemic cardiomyopathy (NICM) $(7,8)$.

In patients with infected cardiac implantable electronic device (CIED) who are still at risk of arrhythmic events, WCD has demonstrated high efficacy and can be used as a bridge until ICD can be re-implanted (9).

A prophylactic ICD implantation among patients after acute myocardial infarction (MI) and high risk of SCD did not reduce overall mortality $(7,10)$. Results of the recent VEST trial showed that WCD did not lead to a significantly lower rate of arrhythmic deaths among patients with a recent MI and LVEF $<35 \%$ (11). ICD implantation for primary prevention is therefore still a difficult topic especially in patients with newly diagnosed NICM (12).

The goal of this study was to compare and analyze differences between less and equal or more than 90 wear days of WCD by looking at (a) baseline characteristics and disease etiology, (b) ICD implantation $v s$. no ICD implantation and (c) improvement of LVEF. 


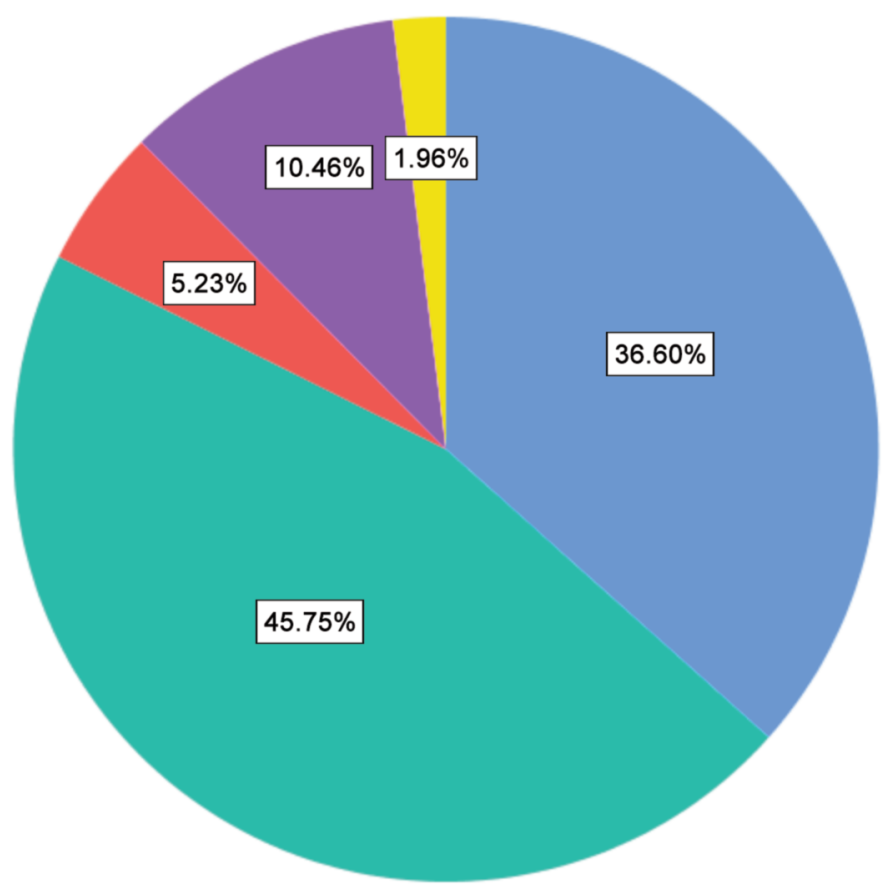

ICM, LVEF $<35 \%$

NICM, LVEF $<35 \%$

Myocarditis

ICD/CRT-D Explantation

congenital diseases

(HCM/Channelopathies)

\section{Patients and Methods}

We included all patients $(\mathrm{n}=153)$ who were prescribed with a WCD between April 2012 and March 2019 at Mannheim University Hospital (Mannheim, Germany). The study was approved by the local ethics committee and conforms to the 1975 Declaration of Helsinki.

WCD is an easy to handle, not invasive and reversible medical device that can detect and terminate ventricular tachycardia (VT) or ventricular fibrillation (VF) $(13,14)$. If an arrhythmic event such as VT or VF occurs, WCD first alarms the patient with vibration and acoustic and optical signals and then treats the patient automatically with up to 5 electric shocks using 150 Joules, if not inhibited by the patient pressing two buttons on the WCD (15). Indications for WCD use were patients at risk of SCD like myocarditis, ICD or cardiac resynchronization therapy (CRT) defibrillator explantation due to infection, newly diagnosed ICM with low LVEF $(<35 \%)$, newly diagnosed non-ischemic cardiomyopathy (NICM) with LVEF $<35 \%$ or congenital diseases (HCM/channelopathies) (Figure 1). Patients received a ZOLL Life Vest TM system (Life Vest, ZOLL, Pittsburgh, PA, USA) and OMT according to current heart failure guidelines (16). In general, WCD was prescribed for 3 months irrespective of the underlying WCD indication described above. Data were prospectively collected. All patients provided consent for collection and analysis of standard clinical data.

Baseline characteristics were obtained at time of heart failure diagnosis and WCD implementation including clinical parameters, medical history and cardiovascular risk factors. We also obtained patient's medication record. Each patient was followed up after 3 months (short-term follow up) and after 6-12 month (long-term follow up) or when clinically indicated. At each examination patient received an electrocardiogram and also an echocardiogram. The biplane Simpson's method was used to calculate LVEF. Improvement was defined as an increase to LVEF over $35 \%$. Reasons for stopping WCD use were either improved LVEF, device implantation, incompliance, death or others.

WCD data were also taken from the manufacturer's online platform (LifeVest Network, ZOLL) including alert records, wear days and daily wear time.

We divided patients into two groups: patients with less than 90 wear days of WCD ( $n=112,<90$ wear days' group) and patients with equal or more than 90 wear days ( $n=41, \geq 90$ wear days' group). After that, we analyzed if patients had ICD implantation or LVEF improvement and compared disease etiology. We used a method similar to the PROLONG study and related a prolongation of WCD wearing days to a) a relevant increase in LVEF with potential to more improvement, especially in cases with LVEF between $30 \%$ to $35 \%$; and b) lack of OMT of heart failure (4).

Each individual alert record, patient-initiated recording or treatment was reviewed and classified into the following categories: appropriate shock, inappropriate shock, inhibition of shock. An arrhythmic episode was divided into sustained VT lasting $30 \mathrm{~s}$ or longer with WCD shock therapy, VF with WCD shock therapy, others with no WCD therapy or none. There were no bradyarrhythmic or asystole events and therefore, they were not categorized in our data table.

Statistical analysis. All statistical analyses were performed using IBM SPSS Statistics for Macintosh (Version 25.0. Armonk, NY, USA: IBM Corp.). Baseline characteristics were compared between patients that used WCD for less than 90 days and $\geq 90$ days. Data are presented as mean \pm standard deviation or median (interquartile range) for continuous variables or as a number of cases for categorical 
Dreher et al: Outcome of Patients Protected by WCD for $<90$ Wear Days versus $\geq 90$ Wear Days

Table I. Baseline characteristics of 153 patients with wearable cardioverter defibrillator (WCD) in comparison of less and equal to or more than 90 wear days.

\begin{tabular}{|c|c|c|c|c|c|}
\hline Variables & & All, $n=153$ & Wear days $<90, \mathrm{n}=112$ & Wear days $\geq 90, n=41$ & $p$-Value \\
\hline \multirow[t]{2}{*}{ Demographics } & Age, mean $\pm \mathrm{SD}$ & $60 \pm 14$ & $60.33 \pm 14$ & $59.63 \pm 12$ & 0.780 \\
\hline & Female, n (\%) & $35(22.9)$ & $27(24.1)$ & $8(19.5)$ & 0.552 \\
\hline \multirow{5}{*}{$\begin{array}{l}\text { Indications for } \\
\text { WCD Use, n (\%) }\end{array}$} & Myocarditis & $8(5.2)$ & $5(4.5)$ & $3(7.3)$ & 0.483 \\
\hline & ICD/CRT-D explant & $16(10.5)$ & $13(11.6)$ & $3(7.3)$ & 0.442 \\
\hline & ICM, LVEF <35\% & $56(36.6)$ & $42(37.5)$ & $14(34.1)$ & 0.974 \\
\hline & NICM, LVEF <35\% & $70(45.8)$ & $49(43.8)$ & $21(51.2)$ & 0.411 \\
\hline & Congenital diseases & $3(2.0)$ & $3(2.7)$ & $0(0)$ & 0.565 \\
\hline \multirow[t]{4}{*}{ Clinic parameter } & Cardiogenic shock at diagnosis, $\mathrm{n}(\%)$ & $13(8.5)$ & $9(8.0)$ & $4(9.8)$ & 0.737 \\
\hline & Pulmonary edema, $\mathrm{n}(\%)$ & $14(9.2)$ & $10(8.9)$ & $4(9.8)$ & 0.876 \\
\hline & Days of hospitalization, mean $\pm \mathrm{SD}$ & $15.78 \pm 11.9$ & $16.34 \pm 13$ & $14.27 \pm 9$ & 0.341 \\
\hline & $\begin{array}{l}\text { Former significant VT/VF prior to } \\
\text { WCD use, } \mathrm{n}(\%)\end{array}$ & $26(17)$ & $19(17.0)$ & $7(17.1)$ & 0.987 \\
\hline \multirow{9}{*}{$\begin{array}{l}\text { Medical history, } \\
\text { n (\%) }\end{array}$} & Former CIED explanted & $7(4.6)$ & $7(6.3)$ & $0(0)$ & 0.006 \\
\hline & History of CAD & $61(39.9)$ & $47(42.0)$ & $14(34.1)$ & 0.385 \\
\hline & History of myocardial infarction & $48(31.4)$ & $34(30.4))$ & $14(34.1)$ & 0.657 \\
\hline & History of CABG & $11(7.2)$ & $9(8.0)$ & $2(4.9)$ & 0.506 \\
\hline & Moderately -severe CKD/Dialysis & $20(13.1)$ & $16(14.3)$ & $4(9.8)$ & 0.465 \\
\hline & History of CHF & $23(15.0)$ & $19(17.0)$ & $4(9.8)$ & 0.224 \\
\hline & History of atrial fibrillation/flutter & $37(24.2)$ & $29(25.9)$ & $8(19.5)$ & 0.418 \\
\hline & History of TIA/stroke & $9(5.9)$ & $8(7.1)$ & $1(2.4)$ & 0.176 \\
\hline & History of COPD & $16(10.5)$ & $12(10.7)$ & $4(9.8)$ & 0.865 \\
\hline \multirow[t]{4}{*}{ Medication, n (\%) } & Betablocker & $147(96.1)$ & $107(95.5)$ & $40(97.6)$ & 0.568 \\
\hline & ACE Inhibitors & $133(86.9)$ & $99(88.4)$ & $34(82.9)$ & 0.083 \\
\hline & Aldosterone antagonists & $87(56.9)$ & $60(53.6)$ & $27(65.9)$ & 0.174 \\
\hline & Diuretics & $105(68.6)$ & $75(67.0)$ & $30(73.2)$ & 0.464 \\
\hline \multirow{6}{*}{$\begin{array}{l}\text { Cardiovascular risk } \\
\text { factors, } \mathrm{n}(\%)\end{array}$} & Smoking & $67(43.8)$ & $54(48.2)$ & $13(31.7)$ & 0.092 \\
\hline & Diabetes mellitus & $31(20.3)$ & $19(17.0)$ & $12(29.3)$ & 0.204 \\
\hline & Overweight $\left(\mathrm{BMI}>25 \mathrm{~kg} / \mathrm{m}^{2}\right)$ & $106(69.3)$ & $75(67.0)$ & $31(75.6)$ & 0.405 \\
\hline & Lipidemia & $67(43.8)$ & $52(46.4)$ & $15(36.6)$ & 0.850 \\
\hline & Hypertension & $87(56.9)$ & $64(57.1)$ & $23(56.1)$ & 0.909 \\
\hline & Family history of CVD & $42(27.5)$ & $31(27.7)$ & $11(26.8)$ & 0.315 \\
\hline
\end{tabular}

ICD: Implantable cardioverter defibrillator; CIED: cardiac implantable electronic device; CRT: cardiac resynchronization therapy; LVEF: Left ventricular ejection fraction; ICM: ischemic cardiomyopathy; NICM: non-ischemic cardiomyopathy; VT: ventricular tachycardia; VF: ventricular fibrillation; CAD: coronary artery disease; CABG: coronary artery bypass graft; CKD: chronic kidney disease; CHF: congestive heart failure; TIA: transient ischemic attack; COPD: chronic obstructive pulmonary disease; BMI: body-mass-index; CVD: cardiovascular disease; CRT-D: cardiac resynchronization therapy-defibrillator; SD: standard deviation.

variables expressed as frequencies and percentages. A paired $t$-test was performed for comparison of continuous variables and a chisquared test was used for comparison of categorical variables. A twotailed $p$-value of $<0.05$ was considered statistically significant.

\section{Results}

WCD Data. A total of 153 patients were prescribed WCD use. Forty-one of these patients $(27 \%)$ used this device for exactly or more than 90 days. Thirty patients ( $73 \%$ of those prescribed WCD $\geq 90$ days) wore the WCD for 90-119 days and 11 patients $(27 \%)$ from 120 to a maximum of 201 days. Median in the $<90$ wear days' group was 48.5 days [interquartile range $(I Q R)=24.25-64]$ and 104 (IQR=94-139.5) days in the other one. The average daily wear time in the $\geq 90$ wear days' group was greater than in the $<90$ wear days' group $(22.38 \pm 2 \mathrm{vs}$. $21.11 \pm 4$ h per day, $p<0.007)$.

Baseline characteristics (Table I). Looking at the indications for WCD use, most of the patients in both groups had a newly diagnosed NICM (43.8\% in the $<90$ wear day's group vs. $51.2 \%$ in the $\geq 90$ wear day's group, $p<0.411)$. Second most frequent indication was newly diagnosed ICM $(37.5 \%$ vs. $34.1 \%, \quad p<0.974)$ followed by ICD/cardiac resynchronization therapy defibrillator (CRT- D) explantation (11.6\% vs. $7.3 \%, p<0.442)$ and myocarditis $(4.5 \%$ vs. $7.3 \%$, $p<0.483)$. There were three patients with channelopathies but only in the $<90$ wear days' group. None of them received an CIED implantation. 
Table II. Comparison of follow up clinical data of patients with $<90$ wear days of the wearable cardioverter defibrillator (WCD) versus $\geq 90$ wear days.

\begin{tabular}{|c|c|c|c|c|}
\hline Variables & & $<90$ wear days $(\mathrm{n}=112)^{1}$ & $\geq 90$ wear days $(n=41)^{1}$ & $p$-Value ${ }^{2}$ \\
\hline \multirow{3}{*}{$\begin{array}{l}\text { Echocardiography data, } \\
\text { mean } \pm \text { SD }\end{array}$} & LVEF at baseline & $29.73 \pm 11$ & $27.10 \pm 10$ & 0.170 \\
\hline & LVEF after 3 months & $38.43 \pm 11$ & $35.33 \pm 12$ & 0.150 \\
\hline & LVEF after 6-12 months & $41.97 \pm 12$ & $41.35 \pm 12$ & 0.789 \\
\hline \multirow[t]{9}{*}{ ECG Data (ms), mean \pm SD } & QRS duration at baseline & $109.43 \pm 31, n=112$ & $110.41 \pm 29, n=41$ & 0.861 \\
\hline & QRS duration after 3 months & $107.72 \pm 28, \mathrm{n}=92$ & $106.84 \pm 26, n=32$ & 0.879 \\
\hline & QRS duration after 6-12 months & $108.15 \pm 28, \mathrm{n}=84$ & $111.81 \pm 28, \mathrm{n}=38$ & 0.518 \\
\hline & QTc duration (ms) at baseline & $460.14 \pm 43, n=112$ & $457.80 \pm 78, \mathrm{n}=41$ & 0.818 \\
\hline & QTc duration after 3 months & $445.05 \pm 41, \mathrm{n}=92$ & $445.71 \pm 42, n=32$ & 0.938 \\
\hline & QTc duration after 6-12 months & $449.12 \pm 39, \mathrm{n}=84$ & $450.38 \pm 42, n=38$ & 0.877 \\
\hline & PQ-interval at baseline & $165.60 \pm 26, n=112$ & $163.60 \pm 27, n=41$ & 0.708 \\
\hline & PQ-interval after 3 months & $170.34 \pm 31, \mathrm{n}=92$ & $167.85 \pm 31, n=32$ & 0.719 \\
\hline & PQ-interval after 6-12 months & $167.68 \pm 28, n=84$ & $158.06 \pm 20, n=38$ & 0.093 \\
\hline \multirow[t]{12}{*}{ ECG rhythm, n (\%) } & Sinusrhythm at baseline & 96/112 (85.7) & $37 / 41(90.2)$ & 0.537 \\
\hline & Sinusrhythm after 3 months & $78 / 92(69.6)$ & $27 / 32(65.9)$ & 0.956 \\
\hline & Sinusrhythm after 6-12 months & $66 / 84(58.9)$ & $33 / 38(80.5)$ & 0.251 \\
\hline & AV-Block at baseline & 9/112 (8.0) & 4/41 (9.8) & 0.749 \\
\hline & AV-Block after 3 months & $5 / 92(4.5)$ & $2 / 32(4.9)$ & 0.865 \\
\hline & AV-Block after 6-12 months & $4 / 84(3.6)$ & $1 / 38(2.4)$ & 0.596 \\
\hline & Pacemaker rhythm at baseline & $4 / 112(3.6)$ & $0 / 41(0)$ & 0.045 \\
\hline & Pacemaker rhythm after 3 months & $6 / 92(5.4)$ & $2 / 32(4.9)$ & 0.957 \\
\hline & Pacemaker rhythm after 6-12 months & $12 / 84(10.7)$ & $3 / 38(7.3)$ & 0.359 \\
\hline & Atrial fibrillation at baseline & $7 / 112(6.3)$ & $4 / 41(9.8)$ & 0.460 \\
\hline & Atrial fibrillation after 3 months & $7 / 92(6.3)$ & $3 / 32(7.3)$ & 0.754 \\
\hline & Atrial fibrillation after 6-12 months & $5 / 84(4.5)$ & $3 / 38(7.3)$ & 0.676 \\
\hline \multirow[t]{6}{*}{ Laboratory values } & Elevated Pro-BNP at baseline, $\mathrm{n}(\%)$ & $60(53.6)$ & $24(58.5)$ & 0.588 \\
\hline & Elevated Pro-BNP after 3 months & $44(39.31)$ & $20(48.8)$ & 0.295 \\
\hline & Elevated Pro-BNP after 6-12 months & $26(23.2)$ & $17(41.5)$ & 0.041 \\
\hline & $\mathrm{BNP}$ at baseline, mean $\pm \mathrm{SD},($ median $)$ & $8,874.57 \pm 31958(2697)$ & $4,877.79 \pm 5298(2950)$ & 0.545 \\
\hline & $\mathrm{BNP}$ after 3 months, mean $\pm \mathrm{SD}$, (median) & $2,241.07 \pm 4103(939)$ & $1,779.45 \pm 2585(862)$ & 0.645 \\
\hline & BNP after 6-12 months, mean \pm SD, (median) & $651.85 \pm 811(334.5)$ & $5,433.53 \pm 14011(720)$ & 0.179 \\
\hline
\end{tabular}

LVEF: Left ventricular ejection fraction; ECG: electrocardiography; BNP: brain natriuretic peptide; AV-Block: atrioventricular-block. ${ }^{1}$ number of patients if not mentioned otherwise; ${ }^{2} p$-value for comparison of $<90$ wear days versus $\geq 90$ wear days.

Follow up clinical data (Table II). There were four patients in the $<90$ wear days' group who had a pacemaker rhythm at baseline $(3.6 \%$ vs. $0 \%$ in the $\geq 90$ wear days' group, $p<0.045)$. After 6-12 months, elevation of Pro-BNP occurred numerically more often in the $\geq 90$ wear days' group than in the other group $(41.5 \% v s .23 .2 \%, p<0.041)$ with a greater median of BNP value (720 vs. 334.5, $p<0.179)$.

WCD arrhythmia events (Table III). We could not find any statistically relevant differences between the two groups looking at arrhythmic events during WCD use. Most of the patients had no arrhythmic events $(93.8 \%$ and $92.7 \%$, $p<0.813)$. There were 4 appropriate shocks in the $<90$ wear days' group and 2 shocks in the $\geq 90$ wear days' group (3.6\% $v s .4 .9 \%, p<0.712)$. Only one of these shocks happened after 90 wear days. This patient was prescribed WCD due to recurrent VTs, but his health status became worse when his knee replacement surgery resulted in infection with decompensated global heart failure and pleural effusions.
LVEF improved from $20 \%$ to only $26 \%$ in the first 3 months, but remained at this level during follow up. It took $159 \mathrm{WCD}$ wear days until ICD implantation was possible. WCD shock occurred after 151 days.

None of the patients in our cohort experienced bradyarrhythmic or asystole events, but one patient was falsely treated with an inappropriate shock. In this event, WCD misinterpreted artefactual voltage fluctuations as VT and therefore, activated its tactile and audible alarms, but the patient was not able to push the response button and therefore, could not save himself from the electric shock.

Figure 2 shows shock events in both groups.

Echocardiographic data. While both groups improved significantly in LVEF during follow up from mean $28.6 \pm 10.15 \%$ at baseline to $36.94 \pm 11.34 \%$ after 3 months $(p<0.001)$ and to $40.96 \pm 12.68 \%$ after $6-12$ months $(p<0.001)$, there were no significant differences between the groups at baseline (<90 wear days: $29.73 \pm 11 \%$ vs. 
Table III. Follow up data of patients after wearable cardioverter defibrillator (WCD) use in comparison of $<90$ wear days versus $\geq 90$ wear days.

\begin{tabular}{|c|c|c|c|c|c|}
\hline Variables & & All, $(\mathrm{n}=153)$ & $<90$ days, $\mathrm{n}=112$ & $\geq 90$ days, $n=41$ & $p$-Value ${ }^{1}$ \\
\hline \multirow[t]{3}{*}{ Wear time of WCD } & Average wear time, hours/day & $21.45 \pm 4$ & $21.11 \pm 4$ & $22.38 \pm 2$ & 0.007 \\
\hline & Wear days, mean \pm SD & $65.1 \pm 42$ & $46.17 \pm 26$ & $116.95 \pm 33$ & $<0.001$ \\
\hline & Wear days, Median (IQR) & $60(30-91)$ & $48.5(24.2-64)$ & $104(94-139.5)$ & $<0.001$ \\
\hline \multirow[t]{3}{*}{ WCD Shocks } & Appropriate shock, n (\%) & $6(3.9)$ & $4(3.6)$ & $2(4.9)$ & 0.712 \\
\hline & Inappropriate shock, n $(\%)$ & $0(0)$ & $0(0)$ & $0(0)$ & \\
\hline & Number of inhibitions, mean \pm SD & $12.28 \pm 33$ & $11.38 \pm 36$ & $14.97 \pm 23$ & 0.575 \\
\hline \multirow{4}{*}{$\begin{array}{l}\text { Arrhythmic episodes } \\
\text { during WCD use, } \\
\text { n }(\%)\end{array}$} & None & $143(93.5)$ & $105(93.8)$ & $38(92.7)$ & 0.813 \\
\hline & Ventricular tachycardia & $5(3.3)$ & $3(2.7)$ & $2(4.9)$ & 0.498 \\
\hline & Ventricular fibrillation & $1(0.7)$ & $1(0.9)$ & $0(0)$ & 0.544 \\
\hline & Others & $4(2.6)$ & $3(2.7)$ & $1(2.4)$ & 0.934 \\
\hline \multirow{6}{*}{$\begin{array}{l}\text { Reason for stopping } \\
\text { WCD use, n }(\%)\end{array}$} & Improved LVEF & $77(50.3)$ & $56(50.0)$ & $21(51.2)$ & 0.894 \\
\hline & CIED implantation & $55(35.9)$ & $37(33.0)$ & $18(43-9)$ & 0.215 \\
\hline & Incompliance & $12(7.8)$ & $11(9.8)$ & $1(2.4)$ & 0.132 \\
\hline & Death $^{2}$ & $2(1.3)$ & $2(1.8)$ & $0(0)$ & 0.380 \\
\hline & Decision pending & $1(0.7)$ & $1(0.9)$ & $0(0)$ & 0.544 \\
\hline & Other reasons & $6(3.9)$ & $5(4.5)$ & $1(2.4)$ & 0.568 \\
\hline \multirow{4}{*}{ Echocardiography data } & Not changed LVEF, n (\%) & $49(32.0)$ & $34(30.4)$ & $15(36.6)$ & 0.465 \\
\hline & Declined LVEF, n (\%) & $3(2.0)$ & $2(1.8)$ & $1(2.4)$ & 0.796 \\
\hline & Improved LVEF in first 3 months, $\mathrm{n}(\%)$ & $67(43.8)$ & $53(47.3)$ & $14(34.1)$ & 0.146 \\
\hline & Improved LVEF after 6-12 months, n (\%) & $34(22.2)$ & $23(20.5)$ & $11(26.8)$ & 0.407 \\
\hline \multirow{5}{*}{$\begin{array}{l}\text { Bundle branch block, } \\
\text { n }(\%)\end{array}$} & None & $108(70.6)$ & $77(68.8)$ & $31(75.6)$ & 0.409 \\
\hline & LBBB & $25(16.3)$ & $18(16.1)$ & $7(17.1)$ & 0.882 \\
\hline & RBBB & $17(11.1)$ & $14(12.5)$ & $3(7.3)$ & 0.366 \\
\hline & Sinus arrest/complete $\mathrm{AV}$-Block & $2(1.3)$ & $2(1.8)$ & $0(0)$ & 0.380 \\
\hline & Fascicular block & $1(0.7)$ & $1(0.9)$ & $0(0)$ & 0.544 \\
\hline \multirow{2}{*}{$\begin{array}{l}\text { Device implantation } \\
\text { after WCD use, } \mathrm{n}(\%)\end{array}$} & Yes & $62(41.7)$ & $42(37.5)$ & $20(48.8)$ & 0.208 \\
\hline & $\mathrm{No}^{2}$ & $87(58.3)$ & $67(59.8)$ & $20(48.8)$ & 0.208 \\
\hline \multirow[t]{4}{*}{ Device type, $\mathrm{n}(\%)$} & None & $87(56.2)$ & $68(60.7)$ & $19(46.6)$ & 0.112 \\
\hline & Transvenous single chamber ICD & $13(8.5)$ & $10(9)$ & $3(7.3)$ & 0.752 \\
\hline & CRT-D & $12(7.8)$ & $7(6.3)$ & $5(12.2)$ & 0.226 \\
\hline & Subcutaneous-ICD & $37(24.2)$ & $25(22.3)$ & $12(29.3)$ & 0.374 \\
\hline \multirow{5}{*}{$\begin{array}{l}\text { Reported shocks post } \\
\text { device implantation, } \mathrm{n}(\%) \\
\text { Arrhythmic episodes post } \\
\text { device implantation, n (\%) }\end{array}$} & Yes & $54(87.1)$ & $102(91.1)$ & $37(90.2)$ & 0.875 \\
\hline & No & $8(12.9)$ & $6(5.4)$ & $2(4.9)$ & 0.906 \\
\hline & Sustained VT & $10(16.1)$ & $7(6.3)$ & $3(7.3)$ & 0.813 \\
\hline & VF & $2(3.2)$ & $2(1.8)$ & $0(0)$ & 0.380 \\
\hline & Non sustained VT & $16(25.8)$ & $12(10.7)$ & $4(9.8)$ & 0.864 \\
\hline \multirow{2}{*}{$\begin{array}{l}\text { Death during mean follow } \\
\text { up period, } \mathrm{n}(\%)\end{array}$} & No & 134 (87.6) & $95(84.8)$ & $39(95.1)$ & 0.087 \\
\hline & Yes & $15(9.8)$ & $14(12.5)$ & $1(2.4)$ & 0.064 \\
\hline \multirow[t]{9}{*}{ Rehospitalization ${ }^{3}, \mathrm{n}(\%)$} & No & $41(26.7)$ & $48(31.3)$ & $5(12.2)$ & $<0.001$ \\
\hline & Yes & $102(66.7)$ & $70(62.5)$ & $32(78.0)$ & 0.071 \\
\hline & Unknown & $10(6.5)$ & $7(6.3)$ & $4(9.8)$ & 0.457 \\
\hline & Cardiovascular cause & $84(54.9)$ & $57(50.9)$ & $27(65.9)$ & 0.100 \\
\hline & Congestive heart failure & $10(6.5)$ & $5(4.5)$ & $5(12.2)$ & 0.087 \\
\hline & Atrial fibrillation & $2(1.3)$ & $1(0.9)$ & $1(2.4)$ & 0.456 \\
\hline & Stroke cause & $6(3.9)$ & $3(2.7)$ & $3(7.3)$ & 0.190 \\
\hline & VT/VF cause & $9(5.9)$ & $7(6.3)$ & $2(4.9)$ & 0.749 \\
\hline & Any other cause & $38(24.8)$ & $27(24.1)$ & $11(26.8)$ & 0.730 \\
\hline
\end{tabular}

LVEF: Left ventricular ejection fraction; LBBB: left bundle branch block; RBBB: right bundle branch block; ICD: implantable cardioverter defibrillator; CRT: cardiac resynchronization therapy; VT: ventricular tachycardia; VF: ventricular fibrillation; SD: standard deviation; IQR: interquartile range; CRT-D: cardiac resynchronization therapy-defibrillator; AV-Block: atrioventricular-block. ${ }^{1} p$-value in comparison of $<90$ wear days versus $\geq 90$ wear days; $22 / 87$ patients died due to septic shock and terminal heart failure, 2/87 denied implantation, 4/153 patients lost to follow up; ${ }^{3}$ over mean follow up of $36.2 \pm 15.6$ months, more than one reason possible.

$27.10 \pm 10 \% \geq 90$ wear days, $p<0.170)$, after 3 months follow up ( $<90$ wear days: $38.43 \pm 11 \%$ vs. $35.33 \pm 12 \% \geq 90$ wear days, $p<0.150)$ and after 6-12 months (<90 wear days: $41.97 \pm 12 \%$ vs. $41.35 \pm 12 \% \geq 90$ wear days, $p<0.789$ ).
In the $<90$ wear days' group LVEF did not change during follow up in 34 patients $v s .15$ patients in the other group (30.4\% vs. $36.6 \%, p<0.465)$, declined in 2 patients $(1.8 \%)$ vs. 1 patient in the $\geq 90$ wear days' group $(2.4 \%, p<0.796)$, 


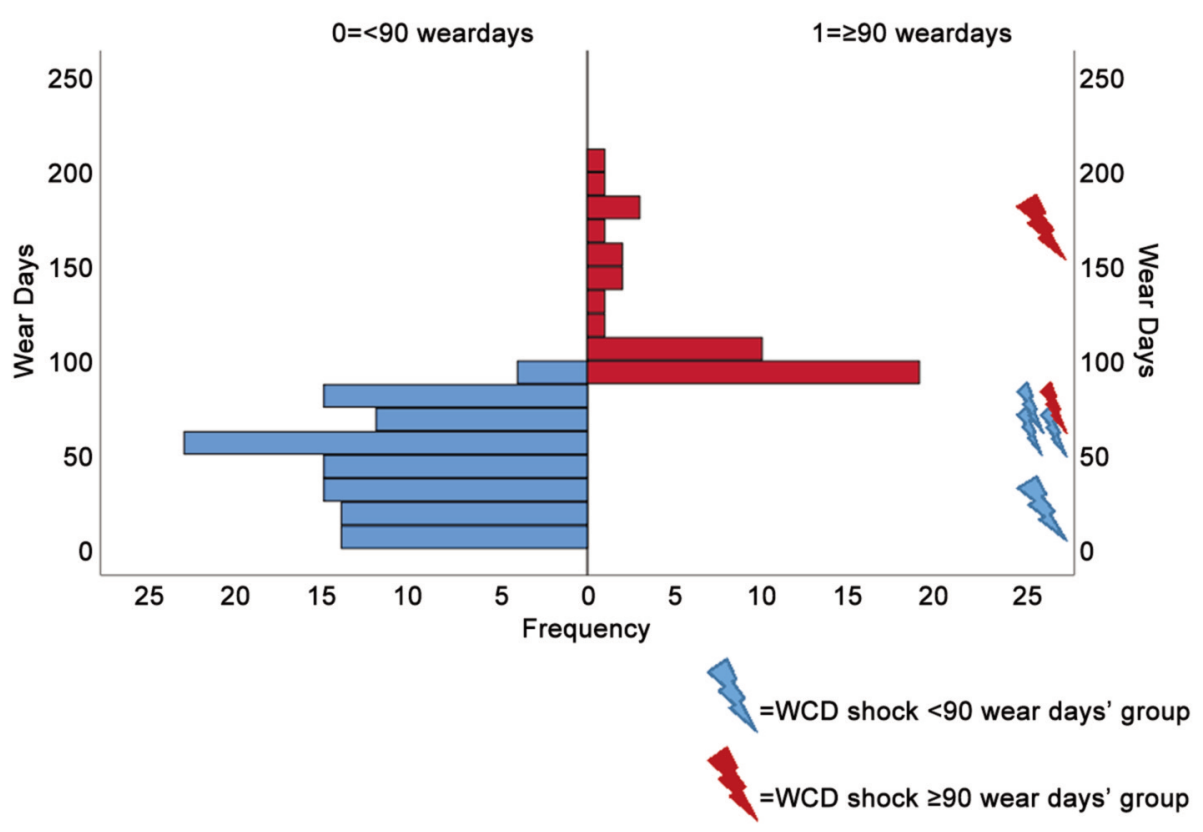

Figure 2. Comparison of wear days and wearable cardioverter defibrillator (WCD) shock therapy.

but improved in 53 patients $(47.3 \%) v s .14(34.1 \%, p<0.146)$ after 3 months and 24 patients $(21.4 \%)$ vs. $11(26.8 \%$, $p<0.407$ ) after 6-12 months.

WCD prolongation outcome (Figure 3). Forty-one out of 153 patients $(26.8 \%)$ had a prolonged WCD use for $\geq 90$ wear days and improved in mean \pm SD LVEF from $27.10 \pm 10 \%$ at baseline to $41.34 \pm 12 \%$ after 6-12 months. Almost half of these patients were diagnosed with NICM $(n=20,48.8 \%)$. Twenty-seven patients did not improve in LVEF after 3 months follow up (65.9\%), but 11 patients did so after 6-12 months $(26.8 \%)$.

Eight patients received ICD implantation because LVEF did not improve during the first 3 months, but improved after 6-12 months when ICD was already implanted. All of them had NICM. In fact, from all 20 NICM patients with extended WCD use, $75 \%$ improved in LVEF either after 3 months $(n=5,25 \%)$ or after $6-12$ months $(n=10,50 \%)$.

Three patients with prolonged WCD wear time improved in LVEF after 6-12 months and did not receive an ICD.

Device implantation. As shown in Figure 3, there is no significant difference in LVEF improvement if the wear days' groups are further divided into no device implantation and device implantation, however, looking at an etiology, there is a higher number of patients with device implantation in the $\geq 90$ WCD wear days' group that were prescribed WCD use because of newly diagnosed NICM than in the device implantation and $<90$ wear days' group $(60 \% v s$. $28.5 \%, p<0.018)$.

ICD events. Six patients from the $<90$ wear days' group with ICD implantation were treated by their device due to VT/VF, and two of them had already received WCD shocks. In the other group, there were two documented VT/VF events during mean follow up, also successfully treated by ICD (14.3\% <90 wear days' group vs. $10 \% \geq 90$ wear days' group, $p=0.638)$.

Death during follow up. Mean follow up time was $36.2 \pm 15.6$ months (median: 34, IQR=24-46 months). Fourteen patients with less than 90 wear days and 1 patient with more than 90 wear days died during this time $(12.5 \%$ vs. $2.4 \%, p<0.064)$. In detail, in the first group 7 patients died without and 7 with ICD implantation. In the $\geq 90$ wear days' group, 1 patient died with device implantation. Reason for death is not known in most of the patients.

Comparison by disease etiology NICM. In detail, there were 69 patients with newly diagnosed non-ischemic cardiomyopathy.

Forty-nine patients with $<90$ WCD wear days $(71 \%)$. Seven patients $(10 \%)$ did not improve in LVEF and either received ICD $(n=4)$ or denied implantation $(n=3)$. Fortytwo patients $(61 \%)$ improved in LVEF after 3 months $(n=29,42 \%)$ or after $6-12$ months $(n=13,19 \%)$. Nine of the 13 patients $(29 \%)$ received device implantation, 


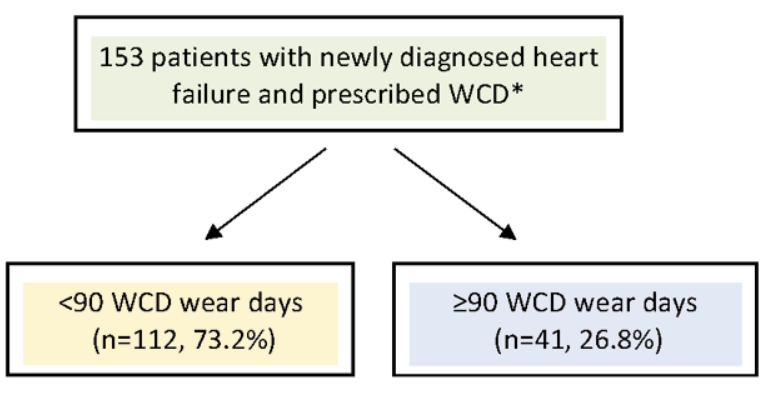

\begin{tabular}{|c|c|c|c|c|c|c|}
\hline & $\begin{array}{c}\text { No } \\
\text { device } \\
\text { Implantation } \\
(n=70,62.5 \%)\end{array}$ & $\begin{array}{c}\text { Device } \\
\text { implantation } \\
(n=42,37.5 \%)\end{array}$ & $\begin{array}{c}\text { No } \\
\text { device } \\
\text { implantation } \\
(n=21,51.2 \%)\end{array}$ & $\begin{array}{c}\text { Device } \\
\text { implantation } \\
(n=20,48.8 \%)\end{array}$ & \multicolumn{2}{|c|}{$p$-Value ${ }^{1}$} \\
\hline $\begin{array}{l}\text { No LVEF } \\
\text { Improvement, } \mathrm{n}(\%)\end{array}$ & $14(20)$ & $20(47.6)$ & $6(28.6)$ & $9(45)$ & 0.405 & 0.861 \\
\hline $\begin{array}{l}\text { LVEF improvement } \\
\text { after } 3 \text { months, } n \\
\text { (\%) }\end{array}$ & $50(71.4)$ & $3(7.1)$ & $12(57.1)$ & $2(10)$ & 0.218 & 0.720 \\
\hline $\begin{array}{l}\text { LVEF improvement } \\
\text { after 6-12 months, } n \\
(\%)\end{array}$ & $6(8.6)$ & $17(40.5)$ & $3(14.3)$ & $8(40)$ & 0.442 & 0.679 \\
\hline $\begin{array}{l}\text { Declined LVEF, n } \\
(\%)\end{array}$ & $0(0)$ & $2(4.8)$ & $0(0)$ & $1(5)$ & & 0.967 \\
\hline Myocarditis, n (\%) & $3(4.3)$ & $2(4.8)$ & $3(14.3)$ & 0 & 0.105 & \\
\hline $\begin{array}{l}\text { ICD/ CRT-D } \\
\text { Explantation, n (\%) }\end{array}$ & $2(2.9)$ & $11(26.2)$ & $2(9.5)$ & $2(10)$ & 0.191 & 0.143 \\
\hline ICM, n (\%) & $25(35.7)$ & $17(40.5)$ & $8(38.1)$ & $6(30)$ & 0.842 & 0.425 \\
\hline NICM, n (\%) & $37(52.8)$ & $12(28.5)$ & $8(38.1)$ & $12(60)$ & 0.235 & 0.018 \\
\hline $\begin{array}{l}\text { Congenital disease/ } \\
\text { channelopathies, n } \\
(\%)\end{array}$ & $3(4.3)$ & $0(0)$ & $0(0)$ & $0(0)$ & & \\
\hline WCD Shocks, $n(\%)$ & $0(0)$ & $4(9.5)$ & $0(0)$ & $2(10)$ & & 0.953 \\
\hline ICD Shock & - & $6(14.3)$ & - & $2(10)$ & & 0.638 \\
\hline $\begin{array}{l}\text { Death during mean } \\
\text { follow up, } n(\%)\end{array}$ & $7(10)$ & $7(16.7)$ & $0(0)$ & $1(5)$ & 0.131 & 0.181 \\
\hline
\end{tabular}

${ }^{1} p$-value for no device implantation $<90$ wear days vs. no device implantation $\geq 90$ wear days, and in comparison with device implantation $<90$ wear days $v s$. device implantation $\geq 90$ wear days;

WCD: Wearable cardioverter/defibrillator; LVEF: left ventricular ejection fraction; ICD: implantable cardioverter defibrillator; CRT: cardiac resynchronization therapy; ICM: ischemic cardiomyopathy; NICM: non-ischemic cardiomyopathy.

Figure 3. Comparison of wear days and device implantation. 
leaving 4 patients with late LVEF improvement, but still without CIED. Four/49 patients from this group died during follow up. One patient with device implantation and one reported successful ICD shock. Reason for death is not known.

We documented 20 WCD patients with $\geq 90$ WCD wear days (29\%). Five of them showed little LVEF improvement, but never reached more than $35 \%$. Four of them were implanted with CIED and 1 patient was lost to long-term follow up. Six patients improved in LVEF after 3 months. One patient with LVEF of $36 \%$ received CRT-D implantation with one reported show during follow up. Nine patients improved in LVEF after 6-12 months (13\%) with 8 of 9 ICD implantations.

From the second group, no patient died during follow up (1 patient lost to follow up). There was 1 patient who received an inappropriate ICD shock due to t-wave oversensing (falsely detected as VT) and 1 patient with documented non-sustained VT during WCD wear time.

\section{Discussion}

Our study shows that a median LVEF improvement occurred in $66 \%$ of patients prescribed WCD. If worn for more than 90 days, more than every fourth patient (26.8\%) improved in LVEF. Therefore, a prolongation of WCD use may prevent patients from inappropriate ICD implantation, but, nevertheless, a longer WCD wear time could imply that patients start to become impatient or bored with a decrease in wear time (hours per day), which could then influence the patient's outcome.

The VEST study showed a mean wear time of $14.0 \pm 9.3 \mathrm{~h}$ in the device group and therefore, WCD did not show a significantly lower rate of arrhythmic deaths in comparison to only medical therapy (11). Subanalysis confirmed that when patients were wearing the WCD, a significantly lower percentage died than when patients did not wear the WCD. This emphasizes the importance of patient's compliance and wear time and that it has direct influence on his or her outcome. A recently published study showed predictors for WCD compliance such as cardiac arrest during myocardial infarction, diabetes or prior heart failure. Whereas factors like divorce, Asian race, high body mass index or WCD shock lead to worse WCD compliance (17). These predictors may be useful in evaluating whether or not to extend WCD use.

Although patients in our cohort with more than 90 wear days had a statistically significant better daily wear time than patients with less than 90 wear days, the rate of arrhythmic deaths in comparison of both groups is not quite statistically significant $(p=0.064)$, but overall survival is high during long-term follow up.

In our cohort, we described patients with newly diagnosed NICM in more detail due to the fact, that prolongation of
WCD wear time in NICM patients is still debatable. Twelve of 69 NICM patients (17\%) did not improve in LVEF during our follow up study. This means that $83 \%(n=57)$ did improve over time. Nevertheless, 27 patients (39\%) already received ICD implantation. All NICM patients with prolonged WCD wear time and ICD implantation had no documented appropriate arrhythmic episodes during follow up.

Two patients that had worn WCD for more than 90 days, without WCD shocks, but subsequently had ICD implantation, showed adequate ICD shock events during follow up and were therefore prevented from SCD. Therefore, WCD might be helpful for risk stratification in patients with an impaired EF.

Every patient who received WCD shock therapy in our cohort was implanted with ICD. During the mean follow up period (36.2 \pm 15.6 months), two of them showed arrhythmic events, such as VT/VF after WCD wear time. Because of ICD implantation, they were successfully treated through defibrillation. Nevertheless, to evaluate whether ICD implantation or a prolonged WCD use is more secure, further randomized and double blinded studies are necessary. Of note, WCD shocks occurred in two patients of the $\geq 90$ wear days' group (1 shock after 151 days), both with ICD implantation after WCD use but without further arrhythmic events during follow up. In addition, three patients with myocarditis and prolonged WCD wear time improved in LVEF and were not implanted with ICD. All 3 survived during mean follow up.

Looking at cost effectiveness, studies have already shown that WCD likely is cost-effective in protecting patients from SCD after removal of infected ICD, while waiting for reimplantation $(18,19)$. In our cohort, further studies need to show if a prolongation of WCD wear time for more than 90 days has a major impact for insurance companies, as long as patients can be at home instead of in a hospital and if a prevention of ICD implantation can result in fewer primary and secondary costs for the health care system.

In addition to a prolongation of WCD wear days there could also be an improvement in OMT. A study has shown that patients with reverse remodeling improved in LVEF due to optimization of heart failure medication (4). Further studies are needed to show whether time, i.e. more than 90 days, or dosage lead to LVEF improvement. Of note, sacubitril-valsartan has shown optimistic effects in heart failure patients (20). In the PARADIGM trial, data have shown that in addition to the lower mortality rate and rehospitalization rate the risk of SCD was decreased. Therefore, testing the concomitant effect of sacubitril/ valsartan with WCD as compared to the historical treatment with ACE-inhibitor and/or AT II antagonists might be a good approach to reduce the number of electronic device implantation, its complications and subsequent costs. 


\section{Limitations}

This study is only a single center study and the number of patients is too small to find significant predictors for LVEF recovery or arrhythmias. Patients were not randomized and there was no control group.

\section{Conclusion}

While two third of our patients recovered from low LVEF to $>35 \%$, still $40 \%$ were implanted with ICD. If WCD was worn for equal to or more than 90 days, every fourth patient recovered from low LVEF. Nevertheless, patients are still at risk of arrhythmic events and should therefore decide carefully whether to prolong WCD wear time or get ICD implantation to prevent them from sudden cardiac death. Overall, prolonging wearing days of WCD for more than 90 days (3 months) does not statistically reduce the number of untimely implanted ICDs.

\section{Conflicts of Interest}

All Authors declare that there are no conflicts of interest regarding this study.

\section{Authors' Contributions}

TD and IE wrote the manuscript and were involved in statistical analysis and design of the study. IE, SR, SLR, SG and JK collected data. IA and MB corrected and revised the manuscript critically. All Authors read and approved the final manuscript.

\section{References}

1 Priori SG, Blomstrom-Lundqvist C, Mazzanti A, Blom N, Borggrefe M, Camm J, Elliott PM, Fitzsimons D, Hatala R, Hindricks G, Kirchhof P, Kjeldsen K, Kuck KH, HernandezMadrid A, Nikolaou N, Norekval TM, Spaulding C, Van Veldhuisen DJ and Group ESCSD: 2015 esc guidelines for the management of patients with ventricular arrhythmias and the prevention of sudden cardiac death: The task force for the management of patients with ventricular arrhythmias and the prevention of sudden cardiac death of the european society of cardiology (esc). Endorsed by: Association for european paediatric and congenital cardiology (aepc). Eur Heart J 36(41): 2793-2867, 2015. PMID: 26320108. DOI: 10.1093/eurheartj/ehv316

2 Piccini JP, Sr., Allen LA, Kudenchuk PJ, Page RL, Patel MR, Turakhia MP, American Heart Association Electrocardiography and Arrhythmias Committee of the Council on Clinical Cardiology and Council on Cardiovascular and Stroke Nursing: Wearable cardioverter-defibrillator therapy for the prevention of sudden cardiac death: A science advisory from the american heart association. Circulation 133(17): 1715-1727, 2016. PMID: 27022063. DOI: $10.1161 / C I R .0000000000000394$

3 Brooks GC, Lee BK, Rao R, Lin F, Morin DP, Zweibel SL, Buxton AE, Pletcher MJ, Vittinghoff E, Olgin JE and
PREDICTS Investigators: Predicting persistent left ventricular dysfunction following myocardial infarction: The predicts study. J Am Coll Cardiol 67(10): 1186-1196, 2016. PMID: 26965540. DOI: $10.1016 /$ j.jacc.2015.12.042

4 Duncker D, Konig T, Hohmann S, Bauersachs J and Veltmann C: Avoiding untimely implantable cardioverter/defibrillator implantation by intensified heart failure therapy optimization supported by the wearable cardioverter/defibrillator-the prolong study. J Am Heart Assoc 6(1), 2017. PMID: 28096098. DOI: 10.1161/JAHA.116.004512

5 Kutyifa V, Moss AJ, Klein H, Biton Y, McNitt S, MacKecknie $\mathrm{B}$, Zareba W and Goldenberg I: Use of the wearable cardioverter defibrillator in high-risk cardiac patients: Data from the prospective registry of patients using the wearable cardioverter defibrillator (wearit-ii registry). Circulation 132(17): 1613-1619, 2015. PMID: 26316618. DOI: 10.1161/CIRCULATIONAHA. 115.015677

6 McMurray JJ, Adamopoulos S, Anker SD, Auricchio A, Bohm M, Dickstein K, Falk V, Filippatos G, Fonseca C, GomezSanchez MA, Jaarsma T, Kober L, Lip GY, Maggioni AP, Parkhomenko A, Pieske BM, Popescu BA, Ronnevik PK, Rutten FH, Schwitter J, Seferovic P, Stepinska J, Trindade PT, Voors AA, Zannad F, Zeiher A, Task Force for the D, Treatment of A, Chronic Heart Failure of the European Society of C, Bax JJ, Baumgartner H, Ceconi C, Dean V, Deaton C, Fagard R, FunckBrentano C, Hasdai D, Hoes A, Kirchhof P, Knuuti J, Kolh P, McDonagh T, Moulin C, Popescu BA, Reiner Z, Sechtem U, Sirnes PA, Tendera M, Torbicki A, Vahanian A, Windecker S, McDonagh T, Sechtem U, Bonet LA, Avraamides P, Ben Lamin HA, Brignole M, Coca A, Cowburn P, Dargie H, Elliott P, Flachskampf FA, Guida GF, Hardman S, Iung B, Merkely B, Mueller C, Nanas JN, Nielsen OW, Orn S, Parissis JT, Ponikowski P and ESC Committee for Practice Guidelines: ESC guidelines for the diagnosis and treatment of acute and chronic heart failure 2012: The task force for the diagnosis and treatment of acute and chronic heart failure 2012 of the european society of cardiology. Developed in collaboration with the heart failure association (hfa) of the esc. Eur J Heart Fail 14(8): 803-869, 2012. PMID: 22828712. DOI: $10.1093 /$ eurjhf/hfs 105

7 Steinbeck G, Andresen D, Seidl K, Brachmann J, Hoffmann E, Wojciechowski D, Kornacewicz-Jach Z, Sredniawa B, Lupkovics G, Hofgartner F, Lubinski A, Rosenqvist M, Habets A, Wegscheider K, Senges J and Investigators I: Defibrillator implantation early after myocardial infarction. N Engl J Med 361(15): 1427-1436, 2009. PMID: 19812399. DOI: 10.1056/NEJMoa0901889

8 Sheppard R, Mather PJ, Alexis JD, Starling RC, Boehmer JP, Thohan V, Pauly DF, Markham DW, Zucker M, Kip KE, McNamara DM and Investigators I: Implantable cardiac defibrillators and sudden death in recent onset nonischemic cardiomyopathy: Results from imac2. J Card Fail 18(9): 675-681, 2012. PMID: 22939035. DOI: 10.1016/j.cardfail.2012.07.004

9 Ellenbogen KA, Koneru JN, Sharma PS, Deshpande S, Wan C and Szymkiewicz SJ: Benefit of the wearable cardioverterdefibrillator in protecting patients after implantable-cardioverter defibrillator explant: Results from the national registry. JACC Clin Electrophysiol 3(3): 243-250, 2017. PMID: 29759518. DOI: 10.1016/j.jacep.2016.09.002

10 Hohnloser SH, Kuck KH, Dorian P, Roberts RS, Hampton JR, Hatala R, Fain E, Gent M, Connolly SJ and Investigators D: 
Prophylactic use of an implantable cardioverter-defibrillator after acute myocardial infarction. N Engl J Med 351(24): 2481-2488, 2004. PMID: 15590950 . DOI: $10.1056 /$ NEJMoa041489

11 Olgin JE, Pletcher MJ, Vittinghoff E, Wranicz J, Malik R, Morin DP, Zweibel S, Buxton AE, Elayi CS, Chung EH, Rashba E, Borggrefe M, Hue TF, Maguire C, Lin F, Simon JA, Hulley S, Lee BK and Investigators V: Wearable cardioverter-defibrillator after myocardial infarction. N Engl J Med 379(13): 1205-1215, 2018. PMID: 30280654. DOI: 10.1056/NEJMoa1800781

12 Haugaa KH, Dan GA, Iliodromitis K, Lenarczyk R, Marinskis G, Osca J, Scherr D and Dagres N: Management of patients with ventricular arrhythmias and prevention of sudden cardiac deathtranslating guidelines into practice: Results of the european heart rhythm association survey. Europace 20(FI2): f249-f253, 2018. PMID: 29878156. DOI: 10.1093/europace/euy112

13 Barraud J, Cautela J, Orabona M, Pinto J, Missenard O, Laine M, Thuny F, Paganelli F, Bonello L and Peyrol M: Wearable cardioverter defibrillator: Bridge or alternative to implantation? World J Cardiol 9(6): 531-538, 2017. PMID: 28706588. DOI: 10.4330/wjc.v9.i6.531

14 Rosenkaimer SL, El-Battrawy I, Dreher TC, Gerhards S, Roger S, Kuschyk J, Borggrefe $M$ and Akin I: The wearable cardioverter-defibrillator: Experience in 153 patients and a longterm follow-up. J Clin Med 9(3), 2020. PMID: 32214048. DOI: $10.3390 /$ jcm 9030893

15 Duncker D and Veltmann C: The wearable cardioverter/ defibrillator - toy or tool? J Atr Fibrillation 8(6): 1367, 2016. PMID: 27909495. DOI: 10.4022/jafib.1367

16 Priori SG, Blomstrom-Lundqvist C, Mazzanti A, Blom N, Borggrefe M, Camm J, Elliott PM, Fitzsimons D, Hatala R, Hindricks G, Kirchhof P, Kjeldsen K, Kuck KH, HernandezMadrid A, Nikolaou N, Norekval TM, Spaulding C and Van Veldhuisen DJ: 2015 ESC guidelines for the management of patients with ventricular arrhythmias and the prevention of sudden cardiac death. Rev Esp Cardiol (Engl Ed) 69(2): 176, 2016. PMID: 26837726. DOI: 10.1016/j.rec.2016.01.001
17 Olgin JE, Lee BK, Vittinghoff E, Morin DP, Zweibel S, Rashba E, Chung EH, Borggrefe M, Hulley S, Lin F, Hue TF and Pletcher MJ: Impact of wearable cardioverter-defibrillator compliance on outcomes in the vest trial: As-treated and perprotocol analyses. J Cardiovasc Electrophysiol 31(5): 10091018, 2020. PMID: 32083365. DOI: 10.1111/jce.14404

18 Jiang X, Ming WK and You JHS: Potential cost-effectiveness of wearable cardioverter-defibrillator for patients with implantable cardioverter-defibrillator explant in a high-income city of china. J Cardiovasc Electrophysiol 30(11): 2387-2396, 2019. PMID: 31502350. DOI: $10.1111 /$ jce. 14153

19 Healy CA and Carrillo RG: Wearable cardioverter-defibrillator for prevention of sudden cardiac death after infected implantable cardioverter-defibrillator removal: A cost-effectiveness evaluation. Heart Rhythm 12(7): 1565-1573, 2015. PMID: 25839113. DOI: 10.1016/j.hrthm.2015.03.061

20 McMurray JJ, Packer M, Desai AS, Gong J, Lefkowitz MP, Rizkala AR, Rouleau JL, Shi VC, Solomon SD, Swedberg K, Zile MR, PARADIGM-HF Investigators and Committees: Angiotensin-neprilysin inhibition versus enalapril in heart failure. N Engl J Med 371(11): 993-1004, 2014. PMID: 25176015. DOI: 10.1056/NEJMoa1409077

Received August 11, 2020

Revised September 7, 2020

Accepted September 9, 2020 